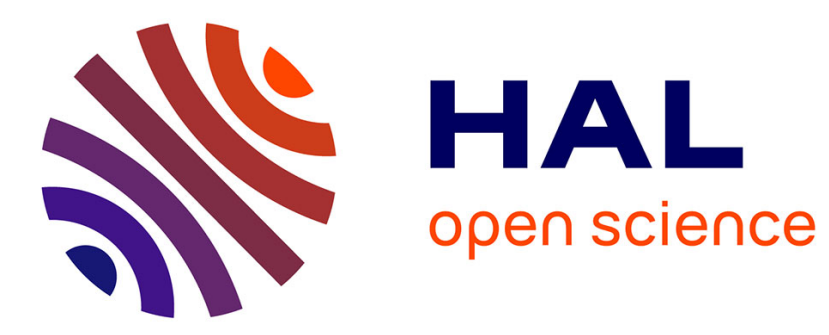

\title{
A Link Reliability Study of Optical Wireless Headset inside Aircraft Cockpit
}

Steve Joumessi-Demeffo, Pierre Combeau, Stéphanie Sahuguede, Damien

Sauveron, Bruno Mercier, Lilian Aveneau, Hervé Boeglen, Anne

Julien-Vergonjanne

\section{To cite this version:}

Steve Joumessi-Demeffo, Pierre Combeau, Stéphanie Sahuguede, Damien Sauveron, Bruno Mercier, et al.. A Link Reliability Study of Optical Wireless Headset inside Aircraft Cockpit. Global Lifi Congress, Jun 2019, Paris, France. hal-02152390

\section{HAL Id: hal-02152390 https://hal.science/hal-02152390}

Submitted on 11 Jun 2019

HAL is a multi-disciplinary open access archive for the deposit and dissemination of scientific research documents, whether they are published or not. The documents may come from teaching and research institutions in France or abroad, or from public or private research centers.
L'archive ouverte pluridisciplinaire HAL, est destinée au dépôt et à la diffusion de documents scientifiques de niveau recherche, publiés ou non, émanant des établissements d'enseignement et de recherche français ou étrangers, des laboratoires publics ou privés. 


\section{A Link Reliability Study of Optical Wireless Headset inside Aircraft Cockpit}

\author{
S. Joumessi-Demeffo, S. Sahuguede, D. Sauveron, \\ A. Julien-Vergonjanne \\ University of Limoges, CNRS, XLIM UMR 7252, \\ F-87000 Limoges, France
}

\begin{abstract}
Communications inside an aircraft cockpit are currently based on wired or radiofrequency connections. For instance, wireless ones have been introduced to support the tablets. However, the use of radiofrequency technologies remains limited. For example, a wireless connectivity for the headset would be an advantage for the pilots in terms of comfort and flexibility but there are some issues especially concerning radiofrequency interferences but also audio data security. Optical wireless communications based on visible light or infrared offer interesting possibilities to overcome these issues. Indeed, as optical beams are confined in the environment, this technology brings robustness against the risks of attacks, thus increasing security. In addition, radiofrequency immunity ensures the absence of disturbances, allowing more resources for communications. For the first time in the literature and using simulation, this paper investigates the optical wireless channel for pilot headset connectivity inside aircraft cockpit, and determines its performance in terms of maximal data rates that can be achieved for a given link reliability.
\end{abstract}

Index Terms - Optical wireless communication; Infrared transmissions; Channel modelling.

\section{INTRODUCTION}

Today, it is accepted that the exponential growth of radiofrequency (RF) communications present a risk of saturation of the currently available spectrum [1]. Different solutions are being explored for future wireless networks. One highly investigated option to solve this issue is the use of the optical spectrum covering the ultraviolet (UV), infrared (IR) and visible bands [2]. Optical Wireless Communications (OWC) are suitable for a wide range of different use-cases offering unique features especially in indoor environments. Indeed, in hospital, industrial or aeronautical scenarios, OWC provide inherent robustness to electromagnetic interference but also hacking of communications [3-6].

Many studies have reported an interest in deploying OWC links in airplanes [7-11]. The related works are generally focused on in-cabin communications because in-flight connectivity can provide new services for passengers. For example, investigated scenarios dealt with in-flight entertainment [7], intra-cabin passenger communication [8], medical monitoring [9] and video streaming [10]. One of the main challenges in airplanes context relies on ensuring an

\author{
P. Combeau, B. Mercier, L. Aveneau, H. Boeglen
}

\author{
University of Poitiers, CNRS, XLIM UMR 7252, \\ F-86000 Poitiers, France
}

adequate cabin environment coverage, requiring a thorough analysis of the communication channel [11]. Actually, many factors have an impact on optical channel quality such as geometry, reflectance properties and positioning of sources and receivers. In addition, significant changes in these parameters due to mobility can affect the channel. These considerations become very important for a new use-case in the aircraft that concerns pilots in the cockpit.

Currently, connections in the cockpit are wired or wireless based on RF. Thanks to the optical wireless technology, it is possible to establish new wireless transmissions, in particular for pilot headset. Indeed, it would bring comfort and mobility to the pilot. In addition, due to confinement of optical beams, this technology is more robust against attacks and therefore can provide a higher level of security. Moreover, due to the frequency band involved in OWC, there is no interference or perturbation with existing RF connections.

We present in this article the study of the optical channel between devices included on the pilot's headset and an access point in the cockpit. As the luminosity within the cockpit must be highly reduced during the critical phases of flight, the visible band is not appropriate in this context, so optical wavelengths in the IR domain are used. In our knowledge, this kind of study is the first one in the literature.

Our work is based on the Monte Carlo ray-tracing methodology, which provides an accurate description of the optical propagation within the specific aircraft cockpit environment. From the $3 \mathrm{D}$ representation of the studied environment, we calculate the received power for Line-OfSight (LOS) and non-LOS (NLOS) paths, i.e. reflected paths over all the elements inside the cockpit. Furthermore, we take into account blockage effects because of body along with head movements of pilots. Based on the channel analysis, we then determine the maximal data rate to guaranty a given link reliability.

The paper is organized as follows. The system description and the optical channel analysis are presented in section 2 . Section 3 focuses on the performance analysis in terms of data rates and reliability. At last, section 4 concludes this paper.

\section{OPTICAL CHANNEL ANALYSIS}

\section{A. System description}

The studied environment is a modern Airbus A350 cockpit as illustrated in Fig. 1 (a). In this context, we focus on a scenario using optical wireless links to ensure the connectivity of 
headsets worn by the aircraft crew (see Fig. 1 (b)). We suppose that at least one member of crew wears the headset continuously during flights. Therefore, it is important to assess link robustness.

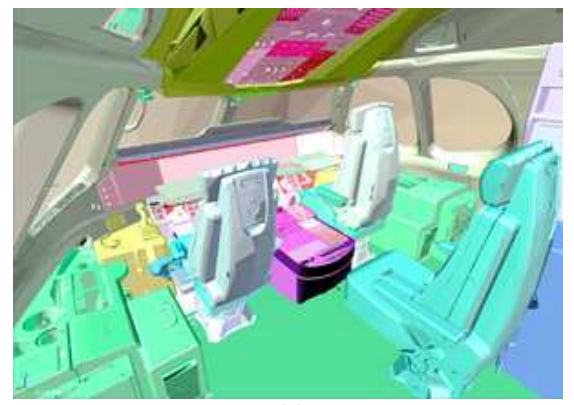

(a)

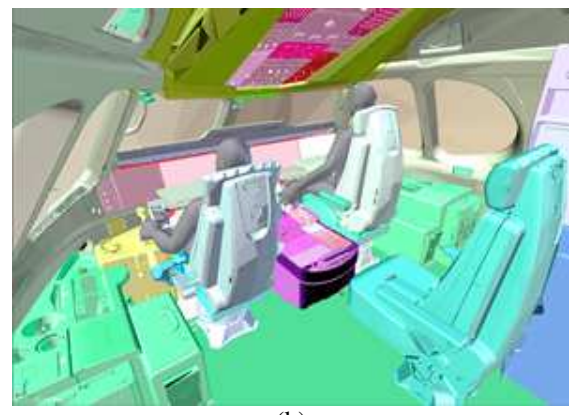

(b)

Fig. 1. (a) 3D view of Airbus A350 cockpit environment; (b) including human bodies

A wireless headset contributes to the comfort of the pilots, especially for long-haul flights. This can be essential especially during operations requiring the pilots to be efficient for example, take-off or landing. As in such critical phases the cockpit lighting is highly reduced, optical communications are studied in the infrared range only.

In the following, we consider IR links between one or several transceivers $\left(\mathrm{T}_{\mathrm{X}} / \mathrm{R}_{\mathrm{X}}\right)$ integrated on the headset and a transceiver located at the cockpit ceiling. The most performing IR configuration is when the emitted optical beams directly falls into the Field-Of-View (FOV) of the receiver, corresponding to a LOS link. However, when using directed sources, LOS links require careful alignment of transceivers, resulting in a small coverage and high sensibility to blockages. In addition, due to headset mobility linked to pilot movements, the LOS condition cannot always be fulfilled. Therefore, NLOS links have to be considered. Thus, in the specific cockpit environment, all the multipath contributions are taken into account, according to the distance between the transceivers, their respective characteristics and orientations, LOS and multi-reflected (NLOS) contributions on cockpit elements, but also pilot and co-pilot presence and movements.

We first investigate a basic uplink between transceivers located on the top of the headsets and a receiver in the cockpit ceiling, named as the access point (AP) and placed on the symmetric axis of the cockpit, at about $1 \mathrm{~m}$ from the top of the pilot's and co-pilot's heads. (see Fig. 2).

We can notice in Fig. 2 that the pilot and co-pilot seats have very different settings. This represents the two limit seat configurations. The pilot is the farthest from the control panel (the face is at $60 \mathrm{~cm}$ ) at the lowest height (the top of the head is $40 \mathrm{~cm}$ under the ceiling and $1.17 \mathrm{~m}$ above the floor). The co-pilot is the closest to the control panel (the face is at $45 \mathrm{~cm}$ ) at the highest height (the top of the head is $15 \mathrm{~cm}$ under the ceiling, $1.30 \mathrm{~m}$ above the floor).

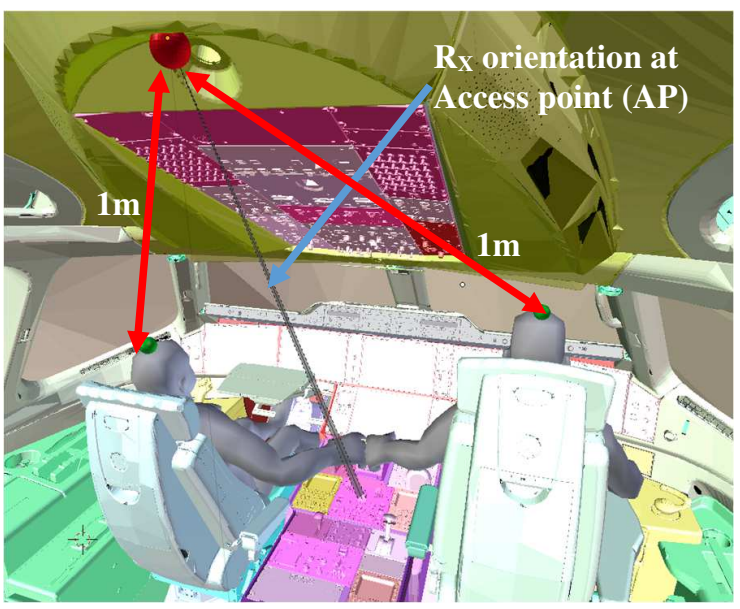

Fig. 2. Basic uplink configuration between emitter on the top of headsets (green spheres) and receiver at AP (red sphere)

We also study the downlink for the same positions, considering that the receiver is either on the pilot or on the copilot headset. In these two configurations, the $\mathrm{T}_{\mathrm{X}}$ orientation at the AP is the same than the $\mathrm{R}_{\mathrm{X}}$ one in the uplink (see Fig. 2). Whatever the link, the pilot and co-pilot bodies are considered as static elements and potential sources of blockages.

To enhance the coverage, it is possible to place several transceivers on the headset, in particular at the ears level. Therefore, we also study the performance because of spatial diversity, considering 5 transceivers on the headset as illustrated in Fig. 3. The one on top of the head is oriented to the ceiling whereas those on each ear are oriented $60^{\circ}$ forward and backward respectively, in order to maximize the coverage.

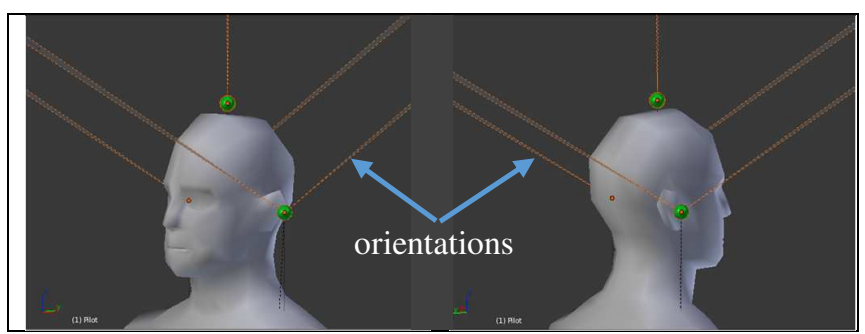

Fig. 3. Transceivers configuration with spatial diversity at the headset

\section{B. Channel modelling}

To determine the impulse response for the different considered channels, we adopt a modeling approach based on a stochastic Monte Carlo method, associated with the ray-tracing algorithm. Our research laboratory has developed the RaPSor software (Ray Propagation Simulator), which is an open source and extensible tool, based on the Netbeans platform for modelling IR and visible links [12,13]. It allows determination of the impulse response $h(\mathrm{t})$ for a defined link.

The following inputs are required for the simulation: 
geometric model and reflection characteristics of the simulation environment materials including pilot's and copilot's bodies, and the definition of key features and locations of the optical transceivers.

The detailed 3D geometric model of the cockpit, composed of $3.3 \mathrm{e} 6$ faces, is first imported into RaPSor. We set the cockpit surface reflection coefficients equal to 0.5 , which is a mean value between absorbent and perfectly reflective materials [13].

In addition to the cockpit itself, important parameters affecting optical propagation are the body and the changes of the transceivers orientation resulting from head movements. The body model representing pilot and co-pilot is a $3 \mathrm{D}$ realistic human mesh of $1.8 \mathrm{~m}$ height and composed of 1004 faces (see Fig. 4). It was fully articulated and animated by using the Blender software in order to take into account a realistic mobility scenario in our channel simulations. As for the cockpit and for the same reason, the reflection coefficient of the body is fixed to 0.5. All the surfaces in the scene are considered as perfectly diffuse and are consequently modeled by a Lambertian Bidirectionnal Reflectance Distribution Function (BRDF) [12].

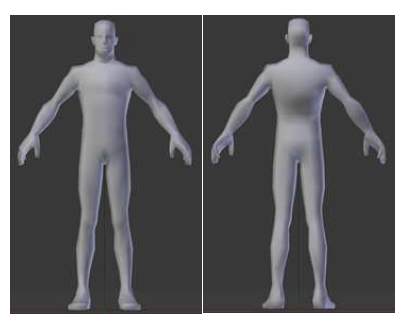

Fig. 4. 3D body model (1004 faces)

The optical characteristics of the transceivers are: type of radiation pattern of the optical source, power spatial distribution of the source defined by the half-power angle $\phi_{1 / 2}$, surface area of the photodetector and FOV.

In the study, we consider Lambertian optical sources with different half-power angles. The receiver is an IR photodiode whose physical area is $7.02 \mathrm{~mm}^{2}$ and having a FOV of $60^{\circ}$.

For a given half-power angle $\phi_{1 / 2}$, we will obtain from simulations a set of impulse responses $h(t)$ corresponding to different IR links according to the orientation changes of headset transceivers induced by the head mobility. The mobility scenario is illustrated in Fig. 5: the pilot first looks ahead and then turns his head to the left, then tilts it forward, and then turns it to the right before returning it to its initial position.

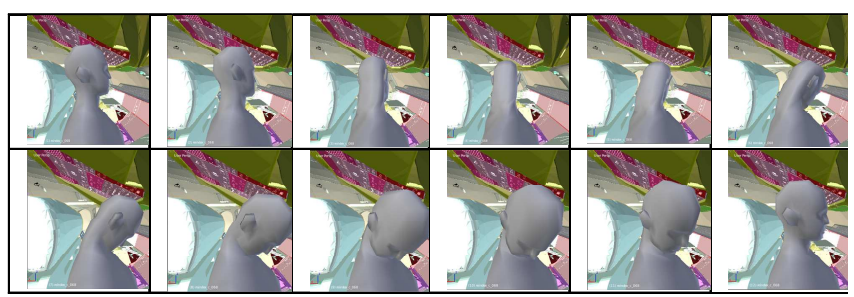

Fig. 5. Illustration of pilot/co-pilot's head movement

Moreover, for all the simulations, we consider a maximum number of 3 successive reflections, which is a classical approach when considering NLOS transmissions and has been checked as being sufficient for simulation convergence.

\section{Channel behavior analysis}

One of the main parameters characterizing the optical channel is the DC gain $H_{0}$, which determines the achievable Signal-to-Noise Ratio (SNR) for a fixed transmitter power [14]. It is defined as:

$$
H(0)=\int_{-\infty}^{+\infty} h(t) d t=H_{0}
$$

where $h(t)$ represents the channel impulse response.

Other features are time dispersion parameters related to impulse responses length and mean delay spreading. The temporal dispersion induced by the reflected paths can be neglected for our use-case (the headset allows audio communications) resulting from the relatively low signal bandwidth. We thus only focus on the DC gain and neglect inter-symbol interference.

The set of impulse responses $h(t)$ resulting from head movements of the pilots leads to a set of optical DC gains $H_{0}$. Therefore, $H_{0}$ is statistically analyzed and channel behavior is characterized by means of the statistical distribution $p\left(H_{0}\right)$.

In this work, we analyze the IR link reliability based on the DC gain cumulative density function $\operatorname{CDF}\left(\mathrm{H}_{0}\right)$ defined as:

$$
\operatorname{CDF}\left(H_{0}\right)=\int_{-\infty}^{H_{0}} p\left(H_{0}\right) d H_{0}
$$

The smaller the $\operatorname{CDF}\left(H_{0}\right)$ for a given $H_{0}$, the higher the reliability.

\section{Results for SISO configurations}

In this part, we first investigate the IR channel behavior for: (a) an uplink between one emitter $\left(\mathrm{T}_{\mathrm{X} 1}\right)$ on the top of the headset and one receiver $\left(\mathrm{R}_{\mathrm{X} 1}\right)$ at the access point, (b) a downlink between $\mathrm{T}_{\mathrm{X} 1}$ at the $\mathrm{AP}$ and $\mathrm{R}_{\mathrm{X} 1}$ on the top of the headset. In each configuration, the analysis is performed with respect to the halfpower angle $\phi_{1 / 2}$ of the emitter $\mathrm{T}_{\mathrm{X} 1}$.

Fig. 6 (a) shows the uplink DC gain $C D F\left(H_{0}\right)$ for the pilot and Fig. 6 (b) the one of downlink channel.

For the uplink, the transmitter orientations vary randomly according to the head movements, whereas the receiver orientation is fixed (since the receiver remains fixed on the cockpit ceiling). It is exactly the inverse case for the downlink. This explains why the curves have a different behavior between the two links.

Concerning the uplink, Fig. 6 (a) shows that the DC gain $\mathrm{CDF}$ is degraded as the half-power angle of the emitter on the headset lowers. As an example, considering a gain value of $70 \mathrm{~dB}$, the CDF value is greater than $40 \%$ for $\phi_{1 / 2}<30^{\circ}$. The CDF is lowered to $20 \%$ for $\phi_{1 / 2}=60^{\circ}$ which appears to be the optimal angle in this configuration.

On the other hand, for the downlink, when head movements induce variations of the receiver orientation, there are cases with strongly attenuated received signal resulting from reflected links and cases of complete blockages. Therefore, channel gain has a larger range of weak values than for the uplink. Fig. 6 (b) shows for the downlink configuration that for a gain value of $70 \mathrm{~dB}$ the best results in terms of CDF are about $60 \%$ obtained for half-power angles $\phi_{1 / 2} \geq 10^{\circ}$. This illustrates that the downlink is less efficient than the uplink. In addition, all 
downlink CDF curves are quite the same and it is difficult to determine an optimal $\phi_{1 / 2}$ value.

Fig. 7 (a) and 7 (b) illustrate the channel gain CDF obtained for the co-pilot position, for the uplink and the downlink respectively. We first notice that, for the co-pilot position, channel gain is degraded compared to the pilot one in particular for the uplink. As an example, for the uplink configuration, Fig. 7 (a) shows that for a gain value of $-70 \mathrm{~dB}$, the lowest CDF value for the co-pilot is around $40 \%$ whereas it was around $20 \%$ for the pilot. Anyway, the optimal half-power angle obtained for the co-pilot is the same that for the pilot for the uplink, i.e. $\phi_{1 / 2}=60^{\circ}$.

Fig. 7 (b) illustrates that for the downlink, channel performance is worse than for the uplink, as it was the case for the pilot position. However, in this case, it is possible to determine an optimal angle around $20^{\circ}$ or $30^{\circ}$.

Nevertheless, the values of uplink or downlink channel gains obtained for both pilot and co-pilot are weak and reliability is not sufficient. Therefore, we investigate spatial diversity to increase channel gain and enhance system communication performance.

\section{E. Results with spatial diversity on the headset}

To enhance the performance mainly affected by the head mobility impact, we propose in this work to use spatial diversity. We consider more precisely five transceivers on the headset as illustrated in Fig. 3.

For the uplink, this means that optical emitted power can be increased because of five identical LED instead of a single one. In addition, as they are located at different positions on the headset, there is less blockage. The access point more easily collects NLOS optical paths, so the DC gain values are improved. Fig. 8 (a) and 9 (a) illustrate this enhancement by comparing the CDFs with those of the basic configuration (without diversity, i.e. Fig. 6 (a) and 7 (a)) also reported for optimal $\phi_{1 / 2}$ values of $60^{\circ}$, for the pilot, respectively the co-pilot. We can first observe that the channel behavior is relatively unchanged regarding the half-power angle of the sources when it is comprised between $30^{\circ}$ and $60^{\circ}$. Therefore, we can conclude that the optimal angle value is still equal to about $60^{\circ}$. In addition, for this optimal angle for the uplink configuration, a CDF value of $20 \%$ is reached for a gain lower than $-55 \mathrm{~dB}$ and $-58 \mathrm{~dB}$ for the pilot, respectively the co-pilot, whereas it was $70 \mathrm{~dB}$, respectively $-75 \mathrm{~dB}$ in the basic configuration. This illustrates the efficiency of diversity at the emitter side for pilot and co-pilot uplinks.

The downlink scenario consists in benefiting of spatial diversity at the receiving side, because of five IR receivers. To study the performance enhancement, we use a basic combining technique which is Selective Combining ( $\mathrm{SwC}$ ) selecting the information of the receiver with the maximal received power [15].

The resulting CDFs of the pilot and co-pilot DC gains are reported in Fig. 8 (b), respectively in Fig. 9 (b) for different half-power angles of the optical source at the access point. In addition, we have also plotted the CDF curves corresponding to the best DC gain values of the basic configuration (without diversity, i.e Fig. 6 (a) and 7 (a)) that is obtained for half-power angles of $30^{\circ}$.
As expected, the first remark is that the channel gain values are improved for the pilot and the co-pilot, especially for an optical emitter with $\phi_{1 / 2}>10^{\circ}$. The proposed spatial diversity on the headset improves the robustness regarding the head movements. In addition, except for $\phi_{1 / 2}=10^{\circ}$ all the curves are quite close, so that we can choose the same angle of $60^{\circ}$ for the AP and headset sources.

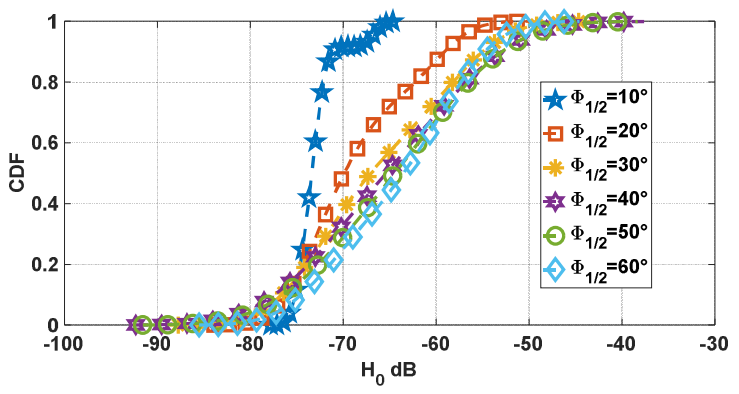

(a)

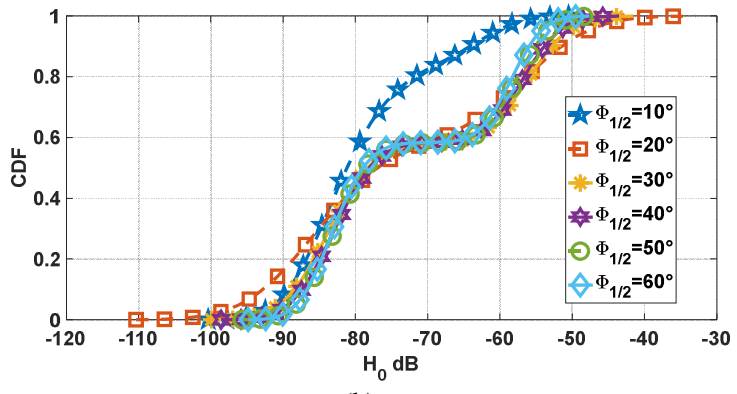

(b)

Fig. 6. CDF of DC channel gain for pilot headset in basic configuration $\mathrm{T}_{\mathrm{X} 1} / \mathrm{R}_{\mathrm{X} 1}$ (a) IR uplink and (b) IR downlink

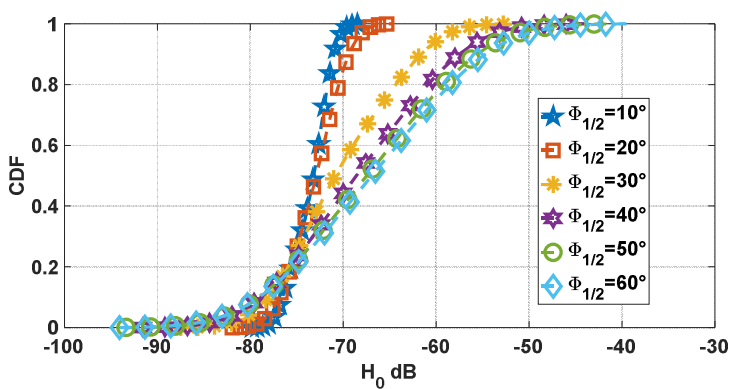

(a)

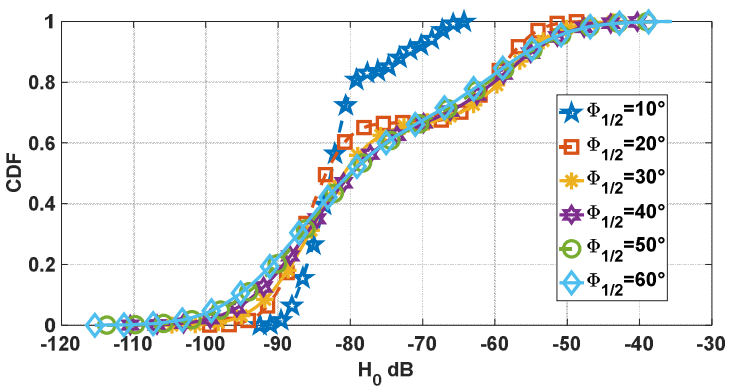

(b)

Fig. 7. CDF of DC channel gain for co-pilot headset in basic configuration $\mathrm{T}_{\mathrm{X} 1} / \mathrm{R}_{\mathrm{X} 1}$ (a) IR uplink and (b) IR downlink

In the following, our aim is to study infrared link reliability for wireless audio transmission in the cockpit. For this purpose, 
we will analyze the case with spatial diversity at the headset side for both pilot and co-pilot, considering five optical sources with $\phi_{1 / 2}=60^{\circ}$ on the headset and one optical source with the same characteristics at the AP.

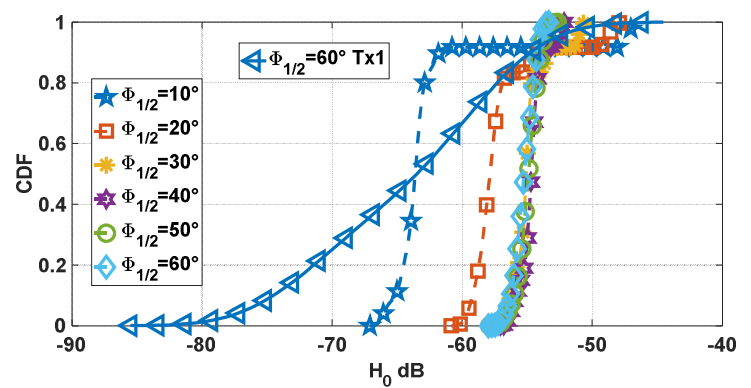

(a)

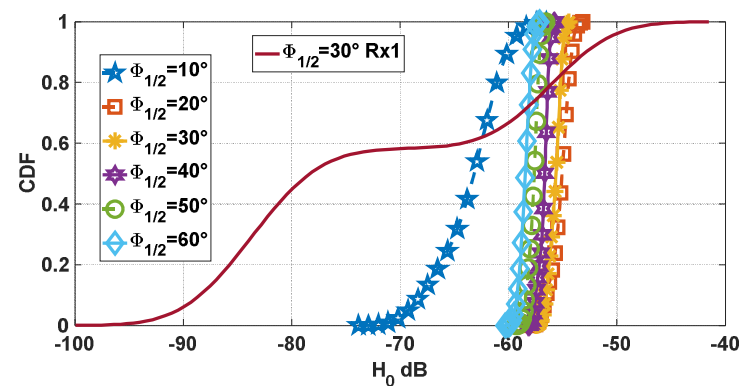

(b)

Fig. 8 CDF of DC channel gain for pilot headset;(a) $5 \mathrm{~T}_{\mathrm{Xi}} / 1 \mathrm{R}_{\mathrm{X} 1}$ IR uplink and (b) $1 \mathrm{~T}_{\mathrm{X} 1} / 5 \mathrm{R}_{\mathrm{Xi}}$ IR downlink

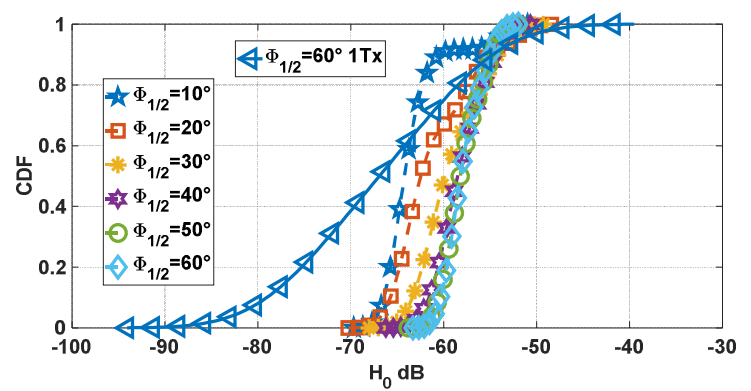

(a)

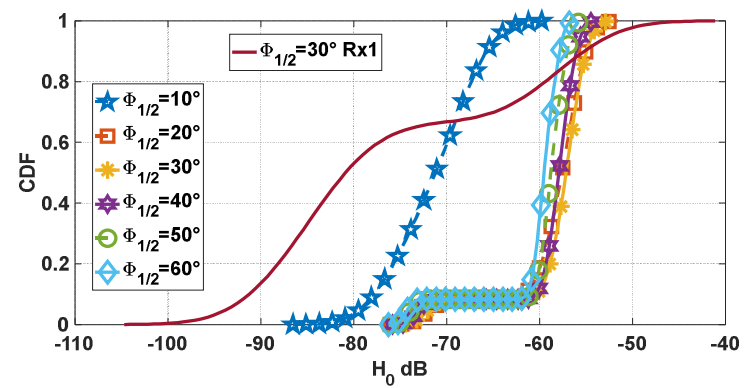

(b)

Fig. 9 CDF of DC channel gain for co-pilot headset; (a) $5 \mathrm{~T}_{\mathrm{Xi}} / 1 \mathrm{R}_{\mathrm{X} 1} \mathrm{IR}$ uplink and (b) $1 \mathrm{~T}_{\mathrm{X} 1} / 5 \mathrm{R}_{\mathrm{Xi}}$ IR downlink

\section{PERFORMANCE ANALYSIS}

\section{A. Definitions}

Linked to the channel DC gain and depending on the system modulation, the Signal to Noise Ratio (SNR) is a classical metric used to evaluate the performance taking into account emitter power and noise contribution. Then, based on SNR, Bit Error Rate (BER) is generally used to establish performance.

However, as $H_{0}$ follows a statistical distribution resulting from head movement as discussed previously, SNR and so BER can be analyzed as random variables. To account for these variations, we use a metric based on the probability of meeting a given performance in terms of BER i.e. a given $\mathrm{BER}_{0}$.

Therefore, we determine the probability that the BER is lower than a target $\mathrm{BER}_{0}$ value, evaluating link reliability. This can be done for different data rates in order to estimate the maximal data rate required to satisfy a given reliability.

\section{B. Performance results}

To illustrate performance, we consider the popular choice for optical wireless systems that is On-Off Keying (OOK) modulation. Considering equiprobable emission and assuming an Additive White Gaussian Noise (AWGN), BER is given by:

$$
B E R=\frac{1}{2} \operatorname{erfc}\left(\sqrt{\frac{S N R}{2}}\right)
$$

where SNR of OOK is expressed as [14] :

$$
S N R=\frac{P_{t}^{2} H_{0}{ }^{2} R^{2}}{4 N_{0} R_{b}}
$$

with $R_{b}$ the data rate, varying parameter in our study, $P_{t}$ the average optical emitted power, $N_{0}$ the noise power spectral density and $R$ the photodiode responsivity set to 1 in the following.

For infrared transmission, we consider that ambient noise is preponderant [14]. Its power is linked to the induced ambient photocurrent $I_{B}$ by:

$$
N_{0}=2 q I_{B}
$$

where $\mathrm{q}$ is the electron quantum charge. We consider in the following typical $I_{B}$ values of $200 \mu$ A for infrared indoor links [16].

Knowing the statistical behavior of $H_{0}$ for a given scenario, we are able to determine the corresponding statistical distribution of the BER for a given data rate, and thus to compute the probability that the BER is lower than a given value named $B E R_{0}$.

We have reported in Fig. 10 an example of results for the downlink pilot scenario with diversity, considering 3 data rate values. The source half-power angle is $60^{\circ}$ and we suppose an average emitted power of $170 \mathrm{~mW}$. We can see in Fig. 10 that for a given data rate, e.g. $10 \mathrm{Mbps}$, the link reliability is of $100 \%$ for BER lower than $10^{-2}$.

To deepen the analysis, we have then determined the maximal data rate guaranteeing a given probability according to the different $\mathrm{BER}_{0}$ values. We conducted this study for the pilot and co-pilot uplink and downlink scenarios with a halfpower angle of $60^{\circ}$. To consider the same emitted power for both links considering diversity on the headset (five sources), the average emitted power per diode for downlink and uplink is $170 \mathrm{~mW}$, respectively $34 \mathrm{~mW}$. Fig. 11 shows the maximal data rates that can be achieved for a reliability of $100 \%$.

We first verify that, as expected for all cases, the maximal data rate $R_{b \text { max }}$ is lowered as the target $\mathrm{BER}_{0}$ diminishes. 
For the pilot, we remark that both links have quite the same performance that is consistent with the results of Fig. 8. We can point out that the data rate values are greater than $1 \mathrm{Mbps}$, which is suitable for an audio application.

On the other hand, the co-pilot curves show that this data rate is not attempted especially in the downlink. For both links the performance for the pilot, outperform always the one for the copilot. As a result, to develop an optical-wireless connected headset in the cockpit reliable regardless of the position of the crew, we must improve the coverage. One way could be to add diversity to the access point in addition to diversity on headsets.

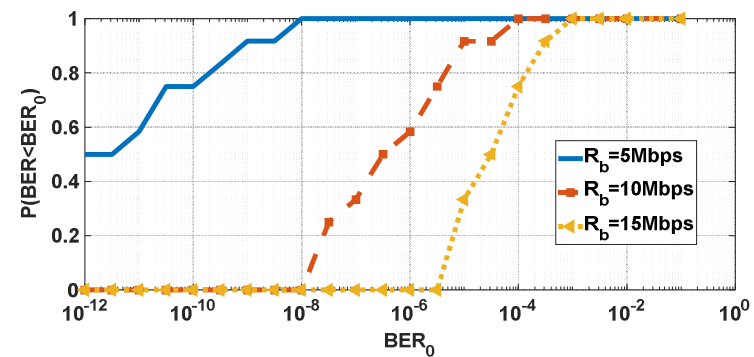

Fig. 10. Probability of BER according target value $\mathrm{BER}_{0}$ for different data rates; case of pilot downlink with source half-power angle of $60^{\circ}$

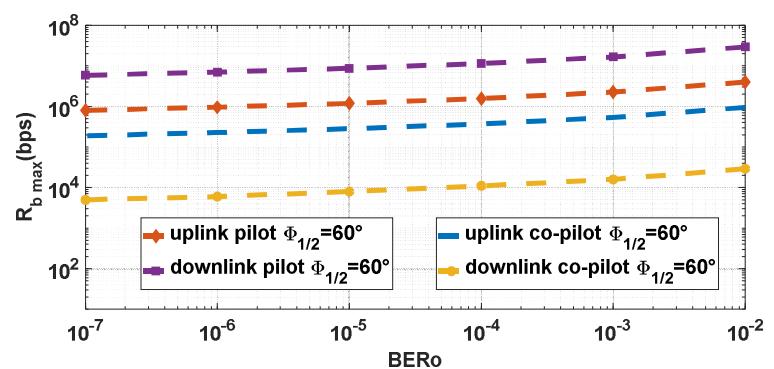

Fig. 11. Maximal data rates for $100 \%$ reliability for uplink and downlink

\section{CONCLUSION}

In this paper, we have presented the study of the optical wireless channel using infrared communication between a headset worn by the crew of an aircraft and an access point located on the ceiling of the cockpit.

The approach was to model the 3D environment of the cockpit and the presence of the pilots thanks to a simulation software based on ray-tracing technique with a Monte-Carlo method. In addition, we integrated the realistic movements of the pilots' heads affecting the propagation of optical rays.

We studied the channel statistical behavior resulting from head movement and related blocking effect, considering pilot and copilot having different sitting positions.

First, a basic uplink and downlink configuration involving a single transceiver on the top of the headset was considered. The analysis of the results allowed determining the optimal source half-power angles for both uplink $\left(60^{\circ}\right)$ and downlink $\left(30^{\circ}\right)$. The results showed that they are identical for the pilot and the co-pilot.

The channel gain and robustness regarding head movements have been then improved using a scenario based on additional transmitters at the ear level of the headset. We have verified that the spatial diversity makes it possible to significantly increase the performance for both links and that the optimal angles are unchanged.

We then evaluated the data rates that can be achieved for a link reliability of $100 \%$ when considering OOK modulation. The results illustrated that an audio application is possible for the pilot sitting position. However, the performance for the copilot sitting position has to be improved, for instance by considering diversity at the AP side.

\section{ACKNOWLEDGEMENT}

The authors would like to thank the European Union and AIRBUS company supporting this work through the Cleansky2 H2020 project named Aircraft Light Communication (ALC).

\section{REFERENCES}

[1] FCC Staff Technical Paper, "Mobile Broadband: The Benefits of Additional Spectrum ," Federal Communications Commission (FCC), Washington, DC, USA, Tech. Rep., October 2010

[2] T. Cogalan and H. Haas, "Why would 5G need optical wireless communications?," 2017 IEEE 28th Annual International Symposium on Personal, Indoor, and Mobile Radio Communications (PIMRC), Montreal, QC, 2017, pp. 1-6.

[3] S. Arnon, J. Barry, G. Karagiannidis, R. Schober, and M. Uysal, Advanced Optical Wireless Communication Systems, 1st ed., NY, USA, Cambridge University Press New York, 2012.

[4] S. Arnon, Visible Light Communication, 1st Ed., NY, USA, Cambridge University Press New York, 2015.

[5] S. Dimitrov and H.Haas, Principles of LED Light Communications Towards Networked Li-Fi., 1st Ed., UK, Cambridge University Press, 2015.

[6] Z. Ghassemlooy, L.N. Alves, S. Zvanovec and M-A Khalighi, Visible Light Communications: Theory and Applications, 1st Ed., FL, USA, CRC Press, Inc. Boca Raton, 2017.

[7] N. Schmitt, Wireless optical NLOS Communication in Aircraft Cabin for In-flight Entertainment, Proc. of ESA 1st Optical Wireless Onboard Communications Workshop, 29-30 Sep. 2004.

[8] C. Vassilopoulos, D. Marinos, A. C. Boucouvalas, N. P. Schmitt, Th. Pistner and C. Aidinis, Diffuse wireless optical link for aircraft intra cabin passenger communication, CSNDSP Proceedings of the $5^{\text {th }}$ International Symposium on Communication Systems Networks and Digital Signal Processing C.3: Optical Wireless Systems, pp. 625-628, July 2006.

[9] D. Marinos, F. Leonidas, N. Vlissidis, C. Giovanis, G. Pagiatakis and C. Aidinis, Medical and safety monitoring system over an incabin optical wireless network, Pages 223-233, February 2011.

[10] S. Dimitrov, H. Haas, M. Cappitelli and M. Olbert, On the throughput of an OFDM-based cellular optical wireless system for an aircraft cabin, Proceedings of the $5^{\text {th }}$ European Conference on Antennas and Propagation (EUCAP), Rome, 2011, pp. 3089-3093.

[11] S. Dimitrov, R. Mesleh, H. Haas, M. Cappitelli, M. Olbert and E. Bassow, Path Loss Simulation of an Infrared Optical Wireless System for Aircrafts, GLOBECOM 2009 - 2009 IEEE Global Telecommunications Conference, Honolulu, HI, 2009, pp. 1-6

[12] A. Behlouli, P. Combeau and L. Aveneau, MCMC Methods for Realistic Indoor Wireless Optical Channels Simulation, Journal of Lightwave Technology, vol. 35, no. 9, pp. 1575-1587, May 2017, 10.1109/JLT.2017.2662939

[13] A. Behlouli, P. Combeau, S. Sahuguède, A. Julien-Vergonjanne, C. Le Bas and L; Aveneau, Impact of Physical and Geometrical Parameters on Visible Light Communication Links, in proc IEEE RTUWO, Riga, Latvia, 2017, pp 73-79.

[14] Z. Ghassemloy, W. Popoola, S. Rajbhandari, Optical wireless communications, system and channel modeling with MATLAB®, $1 \mathrm{st}$ Ed., FL, USA, CRC Press, Inc. Boca Raton, 2012.

[15] A. Goldsmith, Wireless Communications. Cambridge: Cambridge University Press, 2005.

[16] A. J. C. Moreira, R. T. Valadas and A. M. de Oliveira Duarte, Characterization and modelling of artificial light interference in optical wireless communication systems, in proc. of PIMRC, Toronto, Ont., 1995, pp. 326-331. 